\title{
A NEW BATTERY CHARGER/DISCHARGER CONVERTER
}

\author{
R.D.MIDDLEBROOK, SLOBODAN CUK, AND W. BEHEN \\ California Institute of Technology \\ Pasodena, Callfornia
}

\begin{abstract}
A new optimum topology dc-to-dc switchirg converter is extended to provide bidirectional current flow. The resulting two-quadrant converter can be employed to eliminate the discontinuous current mode in normal unidirectional applications, but is especially suited for spacecraft battery conditioning as a charge-discharge regulator in place of the conventional separate converters. Implementation of the control features and the battery charge current and voltage limits are discussed.
\end{abstract}

\section{Introduction}

An extension of a new dc-to-dc switching converter is described, which provides for bidirectional current so that power flow can occur in either direction. The modified converter finds a natural application in a battery charger-discharger regulator, and some system aspects of such an application are discussed together with an experimental circuit.

The new converter, introduced at PESC 1977 [1], is described as optimum-topology because it achieves the general (up or down) dc conversion function with nonpulsating input and output currents with the smallest possible number of elements, namely two inductors, an energy-transfer capacitor, and a singlepole double-throw switch. In its original practical form shown in Fig. 1, the switch is implemented by an NPN transistor and a diode.

In operation, the transistor switch is closed for an interval DT and open for an interval $D^{\prime} T=(1-D) T$, wherre $D$ is the duty ratio and $f \stackrel{s}{s} \mathrm{~T}$ is the switching frequency. During the interval ${ }^{8} \mathrm{D}^{\prime} \mathrm{T}$, the input current passes through the input inductor, coupling capacitor, and diode; the capacitor charges (positively from left to right) with energy delivered from the input and from the

This work was supported in part by the Jet Propulsion Laboratory of the California Institute of Technology, Pasadena, California, under NASA Contract "High Performance Power Systems" (RTOP 506-23-65). inductor. At the same time, the output inductor discharges into the output capacitor and load, and the output current passes through the diode.

Since both inductors are discharging during this interval $D^{\prime} T$ their currents, whose sum passes through the diode, ramps down; if the $D^{\prime} T$ interval terminates before the diode current falls to zero, the diode remains closed until the DT interval begins when the transistor switch is closed. Then, the input current is diverted through the transistor, and the input inductor is recharged. At the same time, the coupling capacitor has its left-hand terminal clamped to ground by the transistor and, since it is already charged positively from left to right, its right-hand terminal is forced negative and the diode is opened. Consequently, the coupling capacitor is discharged into the output capacitor and load and recharges the output inductor, whose current is now diverted through the transistor.

Thus, both the 1nput and output currents flow continuously in a nonpulsating manner, and the pulsating switching current is confined to the
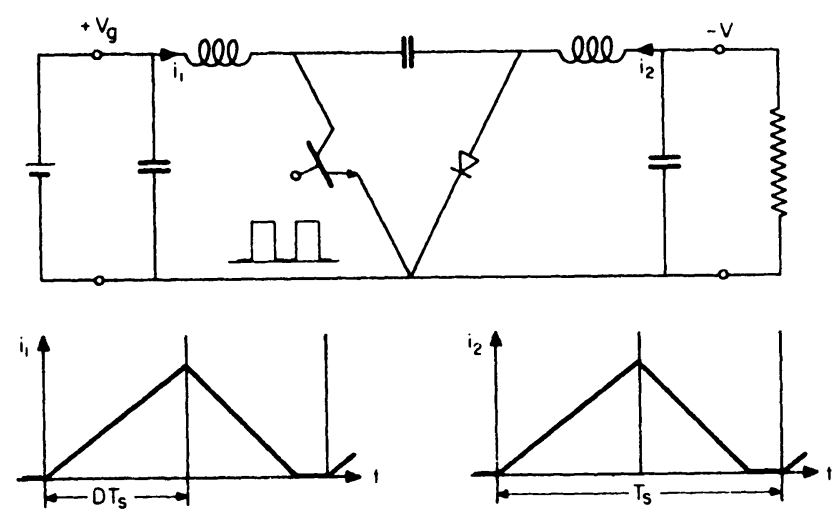

Fig. 1. The original new optimum-topology dc-to-dc switching converter, with input and output inductor current waveforms in the discontinuous diode current mode, for the special case $D=0.5$ for which both inductor currents fall to zero simultaneously. 
Internal loop containing the transistor, coupling capacitor, and diode. This mode of operation, in which the diode current does not fall to zero before the end of the interval $D^{\prime} T$, is usually referred to as the "continuous conduction" mode. The presence of the output capacitor is not essential to the operation of the converter, but is usually included for additional filtering.

\section{Extension to Bidirectional Current Flow}

In the continuous conduction mode, the input and output current waveforms in the original converter of Fig 1 each contain a dc and a ripple component determined by different quantities. The ripple component has an amplitude independent of the load resistance, and the dc component has a value directly dependent on the load resistance. Other conditions constant, an increase of load resistance causes the dc components of the input and output currents, and hence their sum that flows through the diode during the transistor off-time $D^{\prime} T$, to decrease. Also ultimately, since the ripple amplitude is constant, the inverted peaks of the diode current reach zero while the dc component remains greater than zero corresponding to a finite value of load resistance.

This critical value of load resistance describes the boundary between the continuous and discontinuous diode current modes of operation: further increase of load resistance causes the diode current to reach zero before the end of the interval $\mathrm{D}^{\prime} \mathrm{T}$, and the emergence of a third switched circuit conflguration in which both the transistor and diode are open until the beginning of the next DT interval. In the lower part of Fig 1, a special case of discontinuous diode conduction is shown, that for $D=0.5$ in which the input and output inductor currents are equal and so both reach zero at the same time that their sum, the diode current, reaches zero. For other values of $D$, one or the other of the two inductor currents reaches zero first as the load resistance is increased, but discontinuous diode conduction mode does not begin until the first inductor current to reach zero has become negative at the inverted peak to the extent that the sum of the input and output currents is zero.

A thorough analysis of the discontinuous diode conduction mode of the original converter is presented in an accompanying paper [2]. The objective here, in contrast, is to modify the configuration of the converter in such a way that the onset of discontinuous diode current is prevented.

The problem can be solved upon recognition of the cause of the changed mode of operation, namely, that the diode opens when the current through it tries to reverse at the inverted peaks, in deviation from its desired property as an ideal switch. The solution, then, is to install another transistor switch in parallel with the diode to carry the reversed current.
A dual addition is required at the other end of the converter: both the transistor and diode of the original converter carry the sum of the input and output currents during their respective onintervals, so if the diode current tends to reverse so does the transistor current. Therefore, if the diode reverse-current is accommodated by an extra transistor, the original transistor reverse current must be accommodated by an extra diode.

The resulting circult is shown in Fig. 2. It is seen that the configuration is now completely symmetrical with respect to input and output, with the complementary transistor/diode pairs consequent upon the voltage inversion property of the basic converter. An input capacitor is also shown to emphasize the symmetry, but either or both of the input and output capacitors might be omitted.
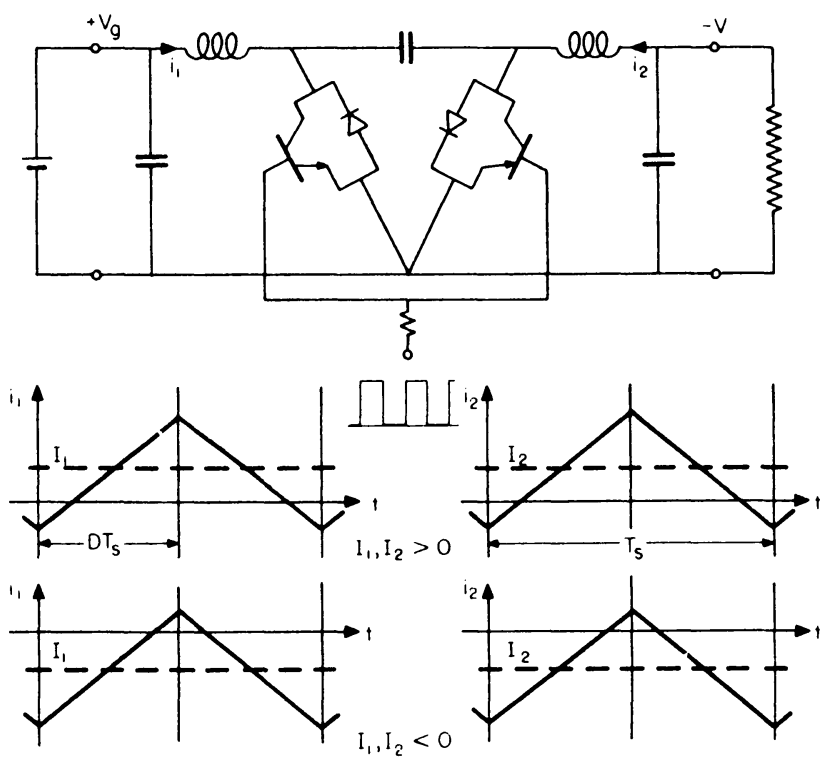

Fig. 2. The extended new converter with symmetrical complementary transistor/diode pairs, which permits bidirectional current flow (twoquadrant operation). The upper waveform diagram shows the two inductor currents in the continuous conduction mode even though the inverted peaks are negative. The lower waveform shows the two inductor currents with negative de components, which could occur if the converter load resistance were replaced with a negative-voltage power source.

The upper waveform diagram in Fig. 2, which again is for the spectal case $D=0.5$, shows how the inverted peaks of the input and output current can go negative, so that the average or dc value of the input or output current can be smaller than in original converter. The load resistance can now become infinite, so that the dc input and output currents can go to zero; consequently, the symmetrical switch configuration of Fig. 2 could be 
employed in a converter to be used in the usual "forward conversion" manner to prevent onset of the discontinuous current mode at light loads. Instead, the continuous current mode would be maintained at all loads, and the otherwise possibly drastic change of ripple magnitudes, gain, and dynamic response would be avolded.

Not only can the output dc current go to zero, but it can in fact reverse. The complete symmetry of the converter circuit in Fig. 2 implies that, with suitable terminations, there is no distinction between "input" and "output:" If the right-hand negative-voltage termination can supply power, the converter dc currents can reverse and supply power to the left-hand termination, as shown in the lower wave form diagrams in Fig. 2, again for the special case of $D=0.5$.

As a result, the circuit of Fig. 2 is bidirectional with respect to current, and is a two-quadrant converter in the sense that, even though the terminal voltage is of fixed polarity, the terminal current can be of either polarity.

This particular result could be achieved in any converter, such as the conventional buck or buckboost, but the bidirectional version of the new optimum-topology converter of F1g. 2 has not only the advantages of the original converter but also the benefit of a particularly simple switch drive. Because both driven switches are referred to a common point, ground, and since the switches are of complementary NPN and PNP type, the two bases can simply be tied together and driven from a single source. Furthermore, overlap is automatically precluded, because even if the drive signal polarity reverses the off transistor cannot be turned on until the stored charge in the base of the on-transistor has been withdrawn, allowing the base polarity to be changed.

Other extensions of the new converter, already described elsewhere, can also be incorporated in the bidirectional version. For example, the input and output inductors can be coupled [3], with resulting ability to "steer" the current ripple towards or away from the input or output, and a single-ended dc isolation transformer can be inserted between the input and output [4].

\section{Battery Charger/Discharger Application}

The two-quadrant bidirectional current version of the new converter is particularly well suited for application as a controlled battery charger and discharger. Its incorporation in a typical spacecraft power system is shown in Fig. 3 .

The basic functional requirement in the system of Fig. 3 is that the battery is to discharge into the loads to maintain the regulated bus voltage when the solar array or radioisotope thermal generator (RTG), or other primary power source, is incapable of supplying the loads. As the output of the primary power source increases, the power supplied by the battery decreases, ultimately to zero. Additional increase in primary source output then goes to recharge the battery.

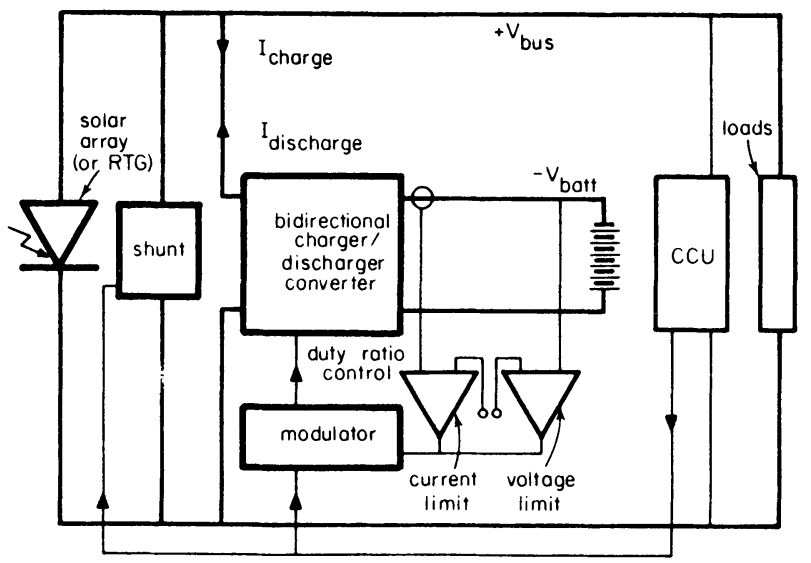

Fig. 3. Incorporation of the two-quadrant converter in a typical spacecraft bus regulator and battery conditioning system. The central control unit (CCU) determines whether the battery is being discharged, on charged subject to current and voltage limits.

System mode of operation is determined by the central control unit (CCU). The CCU provides signals that control the disposition of the array and battery current to maintain bus voltage regulation. At the low end of the bus voltage regulation range, the CCU output provides a signal that causes the modulator to drive the converter switches with a low duty ratio to the NPN transistor, thus allowing the battery to discharge into the bus. As the array power increases less battery power is required, the bus voltage rises, and the CCU signal increases the duty ratio to the converter NPN switch, thus reducing the battery discharge current. As the array output increases further, the same mode of operation continues as the battery current falls to zero when the array power is just sufficient to support the load. Additional increase in array power causes the converter current to reverse, in the source mode of operation, thus initiating battery charge.

Since the battery charge current must be limited, it must be sensed and employed in a local feedback loop at the converter to override the CCU signal and prevent further increase in duty ratio. The overridden CCU control signal then rises further and infitiates conduction of the shunt to absorb the excess array current.

This constant-current battery charging mode continues until the battery voltage reaches a limit sensed by another local voltage feedback loop at the converter, which then overrides the current feedback signal and cuts back the charging current.

Thus, all the necessary battery conditioning functions can be performed by the converter, with addition of the usual programmed or temperaturesensitive current and voltage limits if desired. 
Use of the bidirectional version of the new converter in such a battery charger/discharger system has several advantages. The single converter used for control of charge and discharge precludes the possibility of circulating current that could exist in conventional approaches if the separate charge and discharge regulators operate simultaneously. Also, owing to the basic buckboost conversion function of the new converter, the battery voltage can be efther above or below the bus voltage.

\section{Experimental Implementation of the Battery Charger/Discharger Converter}

An experimental circult has been constructed to fllustrate the battery conditioning subsystem functions. The circuit is shown in Fig. 4, and contains the bidirectional version of the new converter of Fig. 2 plus the local current and voltage limit feedback loops of Fig. 3. The array and battery are simulated by preloaded adjustable power supplies, and the shunt and CCU are omitted.

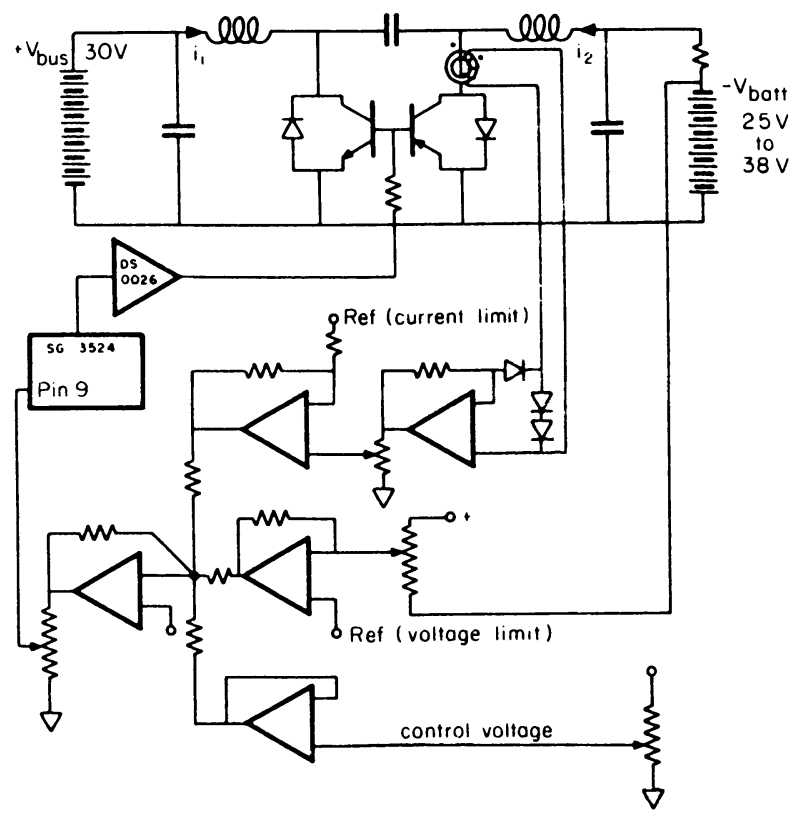

Fig. 4. Experimental test of the two-quadrant converter in a battery conditioning subsystem with current and voltage limits.

The CCU control signal to the converter is replaced by an independent adjustable voltage that can exercise the converter over its full range of operation. The outputs from the independent voltage control amplifier and the current and voltage limit error amplifiers are summed at the comparator input (pin 9) of the Silicon General SG 3524 (whose internal error amplifier is not used), and whose pulse-width - modulated output is amplified by the DS 0026 drivers and applied to the common drive input of the converter switches.

The Fig. 4 subsystem design parameters are: bus voltage $30 \mathrm{~V}$, minimum battery voltage $25 \mathrm{~V}$, battery charge current and voltage limits $2 \mathrm{~A}$ and $35 \mathrm{~V}$. The only feature different from the system block diagram of Fig. 3 is in the battery current sense Implementation.

In principle, as shown in Fig. 3, the battery dc current is to be detected, and if this were implemented directly a dc sense and amplifier would have to be employed. However, the $s$ ame dc value can be detected in an ac manner by the simple expedient of sensing, not the converter output inductor current, but the output diode/transistor current as shown in Fig. 4. This current is pulsating, but has the same average value as does the output inductor current, as shown in Fig. 5, and so can be sensed by a current transformer.

Regardless of the details, it can be seen immediately that the average values of these two currents must be the 8 ame because the only alternative path is through the coupling capacitor, which cannot pass dc. The sense transformer secondary current is amplified by a current-input operational amplifier and core reset is accomplished by the two parallel diodes.

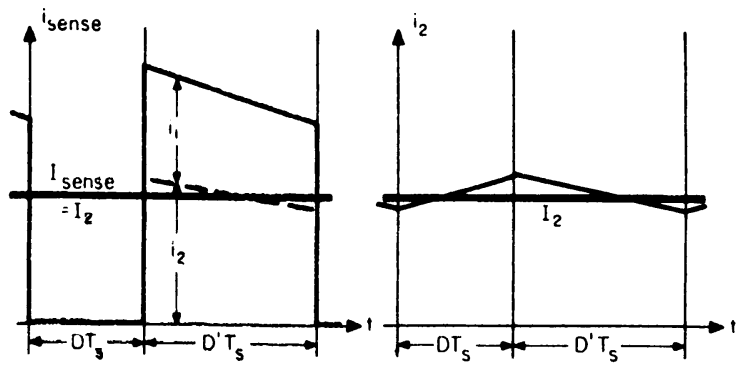

Fig. 5. Battery and PNP/diode current waveforms in the circuit of Fig. 4, showing that their dc components are the same.

The experimental subsystem of Fig. 4 operates according to the above description. It is interesting to note that in practice each transistor switch in the converter actually shares current with the parallel diode by reverse conduction. However, because the reverse current gain is usually low, the transistor reverse conduction soon becomes drive $11 \mathrm{mited}$ and the balance of larger total reverse currents is taken over by the diode.

\section{Conclusion}

By implementation of the ideal switch with complementary transistor/diode pairs, the original optimum-topology dc-to-dc swltching converter has been augmented to a symmetrical form that 18 capable of bidirectional current flow. One 
application of the extended circuit is as a unidirectional converter that maintains the "continuous conduction" mode down to zero dc load current, thus eliminating the severe changes in operational properties normally encountered by entry into the discontinuous conduction mode at low dc load current. Furthermore, only a single drive signal is required for the two switch transistors, and conduction overlap is automatically precluded.

Full advantage is taken of the bidirectional current flow, or two-quadrant properties of the extended new converter in a battery charger/ discharger application. The possibility of simultaneous charge and discharge leading to a circulating current in the conventional approach employing separate charge and discharge regulators is eliminated, with the added benefit of lower parts count. A typlcal spacecraft main bus regulator incoporating the new circult as a battery conditioning converter is described as an application. Implementation of the required battery current and voltage charging limits in our experimental circuit is also described.

All the advantages of the original converter are retalned in the bidirectional extended form, in particular the general dc step-up and step-down capability and the presence of nonpulsating currents at both input and output.

Other extensions that have been described elsewhere, such as coupled input and output inductors and dc isolation, can also be incorporated into the bidirectional version of the optimum-topology converter.
Acknowledgment is made of the work of Shi-Ping Hsu, graduate student in the Power Electronics Group in the Electrical Engineering Department of the California Institute of Technology, who performed some of the experimental work.

\section{REFERENCES}

[1] Slobodan Ćuk and R. D. Middlebrook, "A New Optimum Topology Switching DC-to-DC Converter," IEEE Power Electronics Specialists Conference, 1977 Record, pp. 160-179 (IEEE Publication 77 CH 1213-8 AES).

[2] Slobodan Cuk, "D1scontinuous Inductor Current Mode in the Optimum Topology Switching Converter," IEEE Power Electronics Speciallsts Conference, Syracuse, N.Y., June 13-15, 1978.

[3] Slobodan Cuk and R. D. Middlebrook, "CoupledInductor and Other Extensions of a New Optimum Topology Switching DC-to-DC Converter," IEEE Industry Applications Society Annual Meeting, 1977 Record, pp. 1110-1126 (IEEE Publication 77 CH 1246-8-IA).

[4] R. D. Middlebrook and Slobodan 'Cuk, "Isolation and Multiple Output Extensions of a New Optimum Topology Switching DC-to-DC Converter," IEEE Power Electronics Speclalists Conference, Syracuse, N.Y., June 13-15, 1978. 